\title{
Left Main Stenosis Stenting Normalises Wall Shear Stress of Ascending Aorta in Bicuspid Aortic Valve
}

\author{
Gianluca Rigatelli1 and Marco Zuin'1,2 \\ 1. Cardiovascular Diagnosis and Endoluminal Interventions Unit, Rovigo General Hospital, Rovigo, Italy; 2. University of Ferrara School of \\ Medicine, Ferrara, Italy
}

ntroduction: Bicuspid aortic valve (BAV) is associated with dilation and dissection of the ascending aorta. The high shear forces within the ascending aorta lumen seem to have a pivotal role on the development of such complications. We describe the time-averaged wall shear stress (TAWSS) forces in a patient with normally functioning BAV and significant ostial/mid-shaft left main (LM) stenosis using computational fluid dynamic analysis (CFD). Case report: A 47-year-old female patient with normally functioning BAV with fusion of right and non-coronary cusps was investigated for unstable angina. CFD and stress mapping of the ascending aorta before LM stenting showed a mean TAWSS of 9.4 Pa and was associated with higher TAWSS values at the site of LM stenosis. The LM lesion was treated by stent implantation of an Orsiro (Biotronik, Berlin, Germany) $4.0 \times 12 \mathrm{~mm}$ at $18 \mathrm{~atm}$, preceded with a pre-dilation with non-compliant Euphora (Medtronic Inc., Santa Rosa, CA, USA) balloon $3.0 \times 12 \mathrm{~mm}$ at 16 atm, and followed by an over-dilation with $4.5 \times 12 \mathrm{~mm}$ non-compliant Euphora balloon at 20 atm. The reconstructed post-procedural model revealed a decrease of the mean ascending aorta TAWSS to 5.6 Pa. Conclusions: As suggested by our case, stenting of an LM lesion in a patient with BAV has the potential to improve the TAWSS in the ascending aorta, protecting the ascending aorta from the well-known complications of BAV.

\section{Keywords}

Bicuspid aorta, left main, stenting, angioplasty, wall shear stress

Disclosures: Gianluca Rigatelli and Marco Zuin have no financial or non-financial relationships or activities to declare in relation to this article.

Review Process: Double-blind peer review.

Compliance with Ethics: Informed consent was received from the patient for publication of this case report and accompanying images. No identifying information has been included. Authorship: The named authors meet the International Committee of Medical Journal Editors (ICMJE) criteria for authorship of this manuscript, take responsibility for the integrity of the work as a whole, and have given final approval for the version to be published.

Access: This article is freely accessible at touchCARDIO.com (c) Touch Medical Media 2020

Received: 11 September 2020

Accepted: 18 December 2020

Published Online: 23 December 2020

Citation: Heart International. 2020;14(2):121-2

Corresponding Author: Gianluca Rigatelli,

Cardiovascular Diagnosis and Endoluminal

Interventions, Rovigo General Hospital Viale tre

Martiri, Rovigo 45100, Italy. E: jackyheart@libero.it

Support: No funding was received in

the publication of this article.
Bicuspid aortic valve (BAV) represents the most common congenital cardiac anomaly, with a prevalence ranging between $1 \%$ and $2 \%$ in the general population. ${ }^{1} \mathrm{BAV}$ is known to be associated with dilation and dissection of the ascending aorta, and the significantly higher shear forces seem to have a pivotal role on the development of such complications. ${ }^{2}$ Nowadays, patients with BAV are frequently encountered in clinical practice, presenting coronary artery disease or other comorbidities, due to the increasing survival age of the congenital heart disease population. ${ }^{3}$ While some recent investigations have characterised aortic flow patterns as well as the time-averaged wall shear stress (TAWSS), ${ }^{4-5}$ the aortic arch flow pattern in patients with BAV with a coexisting ostial/mid-shaft left main (LM) stenosis have not been analysed yet. We describe the TAWSS in a patient with normally functioning BAV and significant ostial/mid-shaft LM stenosis before and after LM stenting using computational fluid dynamic analysis (CFD). Informed consent was obtained from the patient and the approval by the local ethics committee was waived due to the type of study.

\section{Case report}

A 47-year-old female patient with normally functioning BAV and fusion of right and non-coronary cusps was investigated for unstable angina. Proximal arteries and aortic arch were reconstructed using coronary computed tomography angiography with a 64-slice multi-detector computed tomography scanner (64-detector row Lightspeed VCT scanner, GE Healthcare, Milwaukee, WI, USA) to define the morphology and degree of dilation of ascending aorta.

The acquired images were then segmented to reconstruct the vascular anatomy in 3D. Coronary artery stenosis and minimal lumen area were calculated considering the reconstructed coronary artery model. Specifically, the severity of the LM stenosis was computed as $100 \%$ minus the percentage of minimal lumen area to the reference lumen area. Blood was modelled as a laminar non-Newtonian and incompressible fluid. Reconstruction and CFD analysis of coronary artery TAWSS were trunked to the proximal segment of the vessels. The aortic wall was assumed to be elastic with a radial dilation of the proximal aorta of $8 \%$ (physiological value around $10 \%$ ) and a total arterial compliance of $1.75 \mathrm{~mL} / \mathrm{mmHg}$ (physiological value $1.84 \pm 0.76 \mathrm{~mL} / \mathrm{mmHg}$ ). ${ }^{3} \mathrm{To}$ estimate the patient-specific flow rate, the patient-specific stroke volume was calculated from the difference between the end-diastolic and end-systolic volumes, and then combined with the measured heart rate to obtain their time-averaged flow rate. Then the calculated velocity was applied as an inlet boundary condition at the left ventricular outflow tract. 


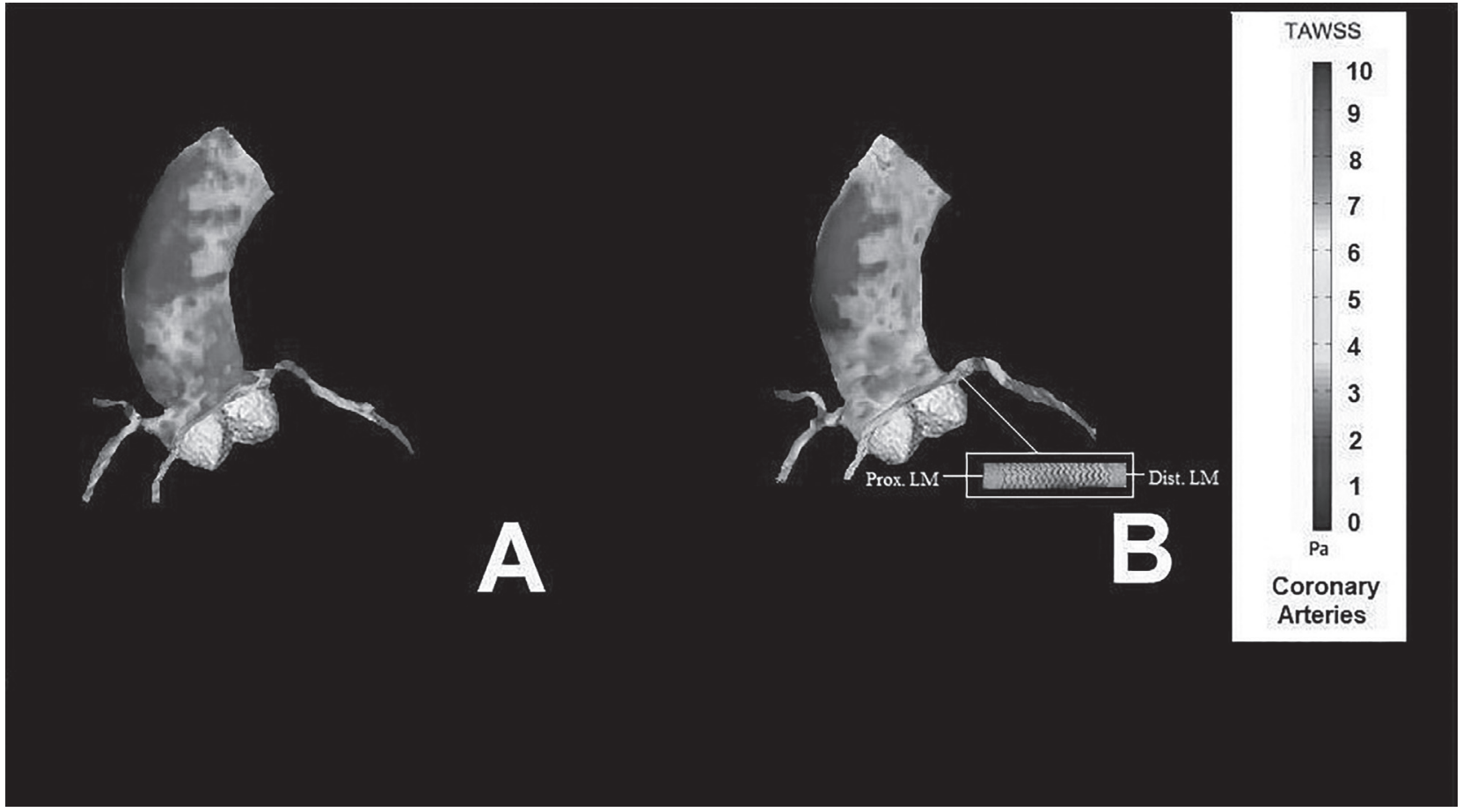

TAWSS showing the higher stress level in the ascending aorta before (A) and after the left main stenting (magnification) (B). Dist. $L M=$ distal left main; TAWSS = time-averaged wall shear stress; Prox. $L M=$ proximal left main .

Stress mapping of the ascending aorta before LM stenting showed a mean TAWSS of $9.4 \mathrm{~Pa}$, and was associated with higher TAWSS values at the site of LM stenosis (Figure 1A). The LM lesion was treated by stent implantation treating the target lesion with an Orsiro (Biotronik, Berlin, Germany) $4.0 \times 12 \mathrm{~mm}$ at $18 \mathrm{~atm}$. Stent implantation was preceded by pre-dilation with non-compliant Euphora (Medtronic Inc., Santa Rosa, CA, USA) balloon $3.0 \times 12 \mathrm{~mm}$ at 16 atm and followed by an over-dilation with $4.5 \times 12 \mathrm{~mm}$ non-compliant Euphora balloon at $20 \mathrm{~atm}$. The reconstructed post-procedural model revealed a decrease of the mean ascending aorta TAWSS to 5.6 Pa.

\section{Discussion}

Our case demonstrated that the treatment of a LM lesion in our patient with BAV improved the TAWSS in the ascending aorta, potentially protecting the ascending aorta from the well-known complications of BAV. Although the association between BAV and LM disease remains very rare in young subjects, the increasing percentage of patients with congenital heart disease reaching middle age and older makes the association between BAV and coronary artery disease more likely to be clinically relevant. ${ }^{3}$

Using 4D flow-sensitive magnetic resonance imaging, Barker et al. demonstrated an increased and asymmetric wall shear stress (WSS) at the aorta wall related to ascending aortic flow jet patterns, which were influenced by the BAV fusion pattern.7 Similarly, Guzzardi et al. observed that regions of increased WSS correlated with extracellular matrix dysregulation and elastic fibre degeneration in the ascending aorta of patients with BAV, suggesting that valve-related haemodynamics may be a contributing factor in the development of aortopathy. ${ }^{8}$

Using CFD analysis in LM disease, our group have already demonstrated that not only a concomitant LM lesion but also the type of stenting significantly modifies the WSS over the ascending aorta. ${ }^{5}$ Extending the stent coverage up to the ostium, when the ostial region is not diseased, might induce unfavourable alterations of flow, not only at the level of the LM lesion and ostium, but also in the ascending aorta and aortic arch, potentially predisposing the aortic wall to long-term damage. In the case of BAV, which already perturbates the aortic arch flow, we hypothesised that the increased thickness of the vessel at the atherosclerotic lesion site may create an additional rigidity point in the aortic wall at the LM site, further worsening the aortic arch flow. This condition, as suggested in the case presented here, can be ameliorated by LM stenting, especially in middle-aged patients where BAV and coronary atherosclerosis can be more frequently associated.

\section{Conclusion}

The treatment of an LM lesion in patients with BAV has the potential to improve TAWSS in the ascending aorta, protecting the ascending aorta from the well-known complications of BAV. Eur Heart J Cardiovasc Imaging. 2013;14:797-804

3. Rigatelli G, Rigatelli G. Congenital heart diseases in aged patients: clinical features, diagnosis, and therapeutic indications based on the analysis of a twenty five-year Medline search. Cardiol Rev. 2005;13:293-6.

4. Keshavarz-Motamed Z, Garcia J, Kadem L. Fluid dynamics of coarctation of the aorta and effect of bicuspid aortic valve. PLOS One. 2013;8:e72394

Rigatelli G, Zuin M, Fong A, et al. Left main stenting induced flow disturbances on ascending aorta and aortic arch. J Trans/ Int Med. 2019;7:22-8.

Rigatelli G, Zuin M, Dell'Awocata F, Nguyen T. Rheolytic effects of left main mid-shaft/distal stenting: a computational flow dynamic analysis. Ther Adv Cardiovasc Dis. 2018; 12:161-8.

Barker AJ, Markl M, Bürk J, et al. Bicuspid aortic valve is associated with altered wall shear stress in the ascending aorta. Circ Cardiovasc Imaging. 2012;5:457-66.

8. Guzzardi DG, Barker AJ, van Ooij P, et al. Valve-related hemodynamics mediate human bicuspid aortopathy: Insights from wall shear stress mapping. J Am Coll Cardiol. 2015;66:892-900. 